\title{
Adipose-derived mesenchymal stem cell therapy in the treatment of knee osteoarthritis: a randomized controlled trial
}

\author{
Julien Freitag*,1,2,3, Dan Bates ${ }^{2,3}$, James Wickham ${ }^{1}$, Kiran Shah ${ }^{3,4}$, Leesa Huguenin ${ }^{2,3}$, Abi \\ Tenen $2,3,5$, Kade Paterson ${ }^{6}$ \& Richard Boyd ${ }^{3,7}$ \\ ${ }^{1}$ School of Biomedical Sciences, Charles Sturt University, NSW, Australia \\ ${ }^{2}$ Melbourne Stem Cell Centre, VIC, Australia \\ ${ }^{3}$ Magellan Stem Cells, VIC, Australia \\ ${ }^{4}$ Faculty of Science, Engineering \& Technology, Swinburne University of Technology, VIC, Australia \\ ${ }^{5}$ Monash University, VIC, Australia \\ ${ }^{6}$ Melbourne School of Health Sciences, The University of Melbourne, VIC, Australia \\ ${ }^{7}$ The Hudson Institute, VIC, Australia \\ *Author for correspondence: Fax: +613 9890 3276; julien.freitag@mscc.com.au
}

Aim: To evaluate the efficacy of autologous adipose-derived mesenchymal stem cell (ADMSC) therapy on pain, function and disease modification in knee osteoarthritis. Methods: 30 participants with symptomatic knee osteoarthritis were randomized into three groups. Two treatment groups received intra-articular ADMSC therapy consisting of either a single injection (100 $\times 10^{6}$ ADMSCs) or two injections $\left(100 \times 10^{6}\right.$ ADMSCs at baseline and 6 months). The third group served as control and continued conservative management. Results: No serious adverse events were observed. Both treatment groups receiving ADMSCs showed clinically significant pain and functional improvement at completion of follow-up at 12 months. Radiological analysis using the Magnetic Resonance Imaging Osteoarthritis Knee Score indicated modification of disease progression. Conclusion: Autologous ADMSC therapy appears to be a safe and effective therapy for knee osteoarthritis and may have the potential to prevent disease progression.

Trial registration number: ACTRN12614000814673

First draft submitted: 8 December 2018; Accepted for publication: 29 January 2019; Published online: 14 February 2019

Osteoarthritis $(\mathrm{OA})$ is an increasingly prevalent and progressive condition causing chronic pain and loss of function. Worldwide it is acknowledged as the fourth leading cause of disability and an important factor affecting disabilityadjusted life years [1,2]. Symptomatic arthritis has a prevalence of $10 \%$ of males and $18 \%$ of females over the age of 45 years with radiological prevalence in up to $80 \%$ of the population at 65 years of age [3-6]. With an aging population this prevalence is expected to increase [3,4].

Conventional conservative medical treatments for knee $\mathrm{OA}$ are aimed at symptomatic control rather than disease modification. These pharmaceutical treatments demonstrate only modest and limited benefits and may have unwanted side effects [7-10]. Surgical total knee replacement, which remains the chosen treatment for symptomatic knee OA that is uncontrolled by conservative therapies, is not without significant complication with as many as $20 \%$ of patients having persistent pain or loss of function at 12 months [11-13].

Whilst historically primary OA has been considered an age-related degenerative condition, we now have a much greater understanding of the pathophysiology of this condition, with an acceptance that OA results due to an imbalance between catabolic/pro-inflammatory and anabolic pathways [14]. Release of reactive oxygen species (free radicals) as a result of mechanical or biological stressors has been shown to lead to chondrocyte cell senescence [15]. This is accompanied by an observed increase in inflammatory cytokine expression including IL-1, TNF- $\alpha$ and matrix metallopeptidase-13 [14-16]. These cytokines further lead to production of other proinflammatory factors, including IL-8, IL-6, leukotriene inhibiting factor, proteases and prostaglandin E2 (PGE2). This host of inflammatory compounds leads to a pro-catabolic state favoring matrix degradation and development of OA. 
The ability of mesenchymal stem cells (MSCs) to differentiate along a mesodermal lineage, including osteoblasts and chondrocytes, has suggested their intrinsic potential in tissue repair and regeneration [17-19]. It is, however, likely that it is via paracrine signaling, rather than direct differentiation that MSCs manipulate the local environment seen in OA, which leads to the disease modification [20].

MSCs are known to have immunomodulatory and anti-inflammatory benefits through suppression of inflammatory T-cell proliferation, inhibition of monocyte maturation and expression of anti-inflammatory cytokines [21-24]. Expression of anabolic cytokines may lead to up-regulation of previously senescent but metabolically active and responsive chondrocytes [25].

Preclinical research has successfully shown benefit of intra-articular MSC therapy in observed pain and functional improvement [26-29]. While disease modification has been observed in some trials, this has not been consistent. Initial clinical trials have encouragingly observed similar pain and functional improvement in symptomatic knee OA following MSC therapy [30-33]. The use of expanded autologous adipose derived MSCs (ADMSCs) in an early Phase I/II trial resulted in radiological evidence of improved cartilage volume and confirmed hyaline-like cartilage on histology [32]. Similarly, the use of allogeneic isolated and expanded bone marrow-derived MSCs had observed improvements in pain and function with improvement in cartilage quality and disease stabilization in comparison to the control group at 12 months [33].

Based upon published clinical trial outcomes, MSC therapy appears safe. In a formal systematic review and meta-analysis of trials involving intravascular administration of either autologous or allogeneic expanded MSC therapies (totaling over 1000 participants) no significant adverse events except transient fever were identified [34]. A systematic review of clinical trials using intra-articular autologous expanded MSC therapies with a mean follow-up of 21 months and assessing 844 procedures similarly showed no association with adverse events including infection, malignancy or death [35].

We present a randomized controlled trial (RCT) to assess the efficacy of autologous ADMSCs in the treatment of symptomatic knee OA. As past studies involving only a single intra-articular MSC injection have postulated potential benefits of multiple injections we have chosen to assess the efficacy of different dosing regimes. While the primary objective is to assess safety, pain and functional changes, a secondary objective is to determine the potential of MSC therapy to achieve disease modification, as detected through radiological examination using magnetic resonance imaging (MRI) techniques.

\section{Materials \& methods}

\section{Trial design}

This RCT was approved by the Human Research Ethics Committee (HREC) of Monash University and registered on the Australian and New Zealand Clinic Trial Registry (Trial ID: ACTRN12614000814673). All participants completed written informed consent. The study was a single centred trial.

The trial design consisted of a total of 30 participants randomly and equally allocated to a control group and two treatment groups. Participants were not blinded to their treatment allocation.

Treatment groups were to receive different treatment dosing regimes of autologous ADMSCs:

- Control: Ongoing conventional conservative management only

- One-injection group: a single intra-articular injection of $100 \times 10^{6}$ ADMSCs

- Two-injection group: two intra-articular injections of $100 \times 10^{6}$ ADMSCs (baseline and 6 months).

A third treatment group receiving five injections of $40 \times 10^{6}$ ADMSCs at baseline, 1, 2, 3 and 6 months, respectively, was intended but ceased due to observed and reproducible moderate adverse events in a concurrently run study with the same treatment protocol (documented as increasing self-limiting pain with sequential injections at monthly intervals) [36]. Participants that were already randomly allocated to this treatment group and yet to commence the trial were re-randomized to another trial group. The HREC of Monash University were informed of this trial protocol variation.

Those participants allocated to the control group maintained their pre-existing conventional conservative management program consisting of simple analgesia, weight management and exercise. Participants were not prescribed a trial-specific conservative program. Participants recorded any changes in their conservative management program. 
Table 1. Eligibility criteria.

\begin{tabular}{|c|c|}
\hline \multicolumn{2}{|c|}{ Inclusion criteria } \\
\hline 1. & Radiological diagnosis of osteoarthritis using the American College of Rheumatology criteria \\
\hline 2. & Radiological grading of Grade II-III osteoarthritis (OA) of the knee as determined by a qualified radiologist using the Kellgren and Lawrence system \\
\hline 3. & Medial or lateral compartment OA as determined above \\
\hline 4. & $\begin{array}{l}\text { Conservative OA treatment already undertaken defined as: analgesia/anti-inflammatory medication, supplements approved by the treating clinician } \\
\text { (e.g., glucosamine sulphate), an attempted exercise program prescribed by a physiotherapist or medical practitioner for at least } 8 \text { weeks, weight loss and } \\
\text { nutritional management as prescribed by a dietitian or medical practitioner for at least } 8 \text { weeks, and biomechanical management including bracing if } \\
\text { appropriate as prescribed by a physiotherapist, podiatrist or medical practitioner. Autologous MSC is an invasive treatment and guidelines recommend } \\
\text { trialing conservative measures as the first line of treatment in knee OA }\end{array}$ \\
\hline 5. & A minimum pain score of 5 on an 11-point numerical rating scale \\
\hline 6. & Single knee osteoarthritis \\
\hline 7. & Less than 5 degrees varus or valgus knee deformity as measured by the long mechanical axis of the knee on $x$-ray \\
\hline 8. & $\begin{array}{l}\text { Sufficient English skills to complete the questionnaires required for the study, as well as to understand the instructions given by the study doctors. This is } \\
\text { required as no funding is available for translation or interpreters, and the outcome questionnaires to be used have only been validated in English language }\end{array}$ \\
\hline \multicolumn{2}{|c|}{ Exclusion criteria } \\
\hline 1. & Pregnancy (accepted contra-indication as no safety data on this population) \\
\hline 2. & Breast feeding (accepted contra-indication as no safety data on this population) \\
\hline 3. & $\begin{array}{l}\text { Have other causes of their knee symptoms suspected to be due to serious pathology such as tumors or referral from the hip or lumbar spine. These } \\
\text { conditions are not under investigation within the current project }\end{array}$ \\
\hline 4. & Bleeding disorder - i.e., hemophilia (accepted contra-indication as no safety data on this population) \\
\hline 5. & MRI confirmed displaced meniscal tear \\
\hline 6. & MRI confirmed Grade IV chondral loss \\
\hline 7. & Previous meniscectomy/significant partial meniscectomy or other knee related surgery within the last 12 months \\
\hline 8. & Previous intra-articular injectable therapies within the last 6 months \\
\hline 9. & History of cancer \\
\hline 10. & History of atypical chronic pain syndrome - i.e., chronic regional pain \\
\hline 11. & History of systemic illness or significant organ impairment/failure (i.e., renal failure) \\
\hline 12. & History of allergy to any substances used within the treatments \\
\hline 13. & $\begin{array}{l}\text { Plans at the time of enrollment to undergo surgery in the following } 12 \text { months. This criterion is aimed at avoiding co-interventions that may confound the } \\
\text { results of the study. While involvement in the project will not strictly prevent participants from undertaking such interventions if required, we will exclude } \\
\text { volunteers who already have such procedures scheduled }\end{array}$ \\
\hline
\end{tabular}

\section{Participants}

Eligible participants 18 years or older with unilateral moderate symptomatic knee pain and Grade II-III medial or lateral compartment knee OA according to Kellgren Lawrence criteria were recruited. All participants had previously attempted primary conservative management of OA including simple analgesia, an exercise and weight management program and biomechanical adjustment with bracing or orthotics if relevant. Formal eligibility criteria is outlined in Table 1.

Baseline assessment involving a subjective and abbreviated musculoskeletal physical examination was performed with diagnostic $x$-ray and MRI assessed to determine eligibility criteria as indicated in Table 1. Participants received formal written information about the requirement of involvement in the trial and were invited to provide written informed consent.

\section{Intervention}

Autologous ADMSC preparation

Adipose tissue was chosen as the source of autologous MSCs due to the relative ease of harvest, abundance of MSCs and shared chondrogenic potential in comparison to other source sites such as bone marrow [37-39].

The harvest, isolation and expansion of ADMSCs has been previously published [36,40,41]. Participants randomly allocated to a treatment group underwent an abdominal lipoharvest procedure. Using a lateral abdominal approach, the subcutaneous fat was infiltrated with up to $300 \mathrm{ml}$ of tumescent fluid (comprising of $30 \mathrm{ml}$ of $2 \%$ lidocaine, $1 \mathrm{ml}$ of $1: 1000$ adrenaline and $1 \mathrm{ml}$ of $8.4 \%$ bicarbonate suspended in a normal saline solution to a total $1000 \mathrm{ml}$ ). Following this, up to $60 \mathrm{ml}$ of adipose tissue and tumescent fluid was aspirated through a $4 \mathrm{~mm}$ lipoaspirate cannula 
Table 2. Fluorescence-activated cell sorting surface marker analysis. Mean data $(n=20)$ is presented with standard deviation in brackets.

\begin{tabular}{|c|c|c|c|c|c|c|c|}
\hline & \multicolumn{3}{|c|}{ Histograms - positive markers } & \multicolumn{4}{|c|}{ Histograms - negative markers } \\
\hline & CD90+ve & CD73+ve & CD105+ve & CD14+ve & CD19+ve & CD34+ve & CD45+ve \\
\hline Percentage & $98.17(2.4)$ & $97.75(3.1)$ & $93.78(6.6)$ & $0.89(1.0)$ & $0.31(0.4)$ & $0.95(1.1)$ & $0.63(0.7)$ \\
\hline
\end{tabular}

Table 3. Average cell count and viability confirmed using a muse cell analyzer.

Cell count (mean \pm standard deviation) Viability (mean \pm standard deviation)

\begin{tabular}{|llll}
\hline One-injection group & Baseline injection & 103.9 million (7.7) & $95.4 \%(2.3)$ \\
\hline Two-injection group & Baseline injection & 95.1 million (11.1) & $93 \%(4.2)$ \\
\hline & 6 month injection & 102.6 million (8.3) & $92.9 \%(3.6)$
\end{tabular}

and collected within a sterile medical grade single use Shippert Tissu-Trans Collection filter (Shippert Medical, CO, USA).

The lipoaspirate was transferred directly to an onsite clean room laboratory facility for later isolation and expansion of autologous ADMSCs (Magellan Stem Cells, Melbourne, Australia). All tissue processing was performed within a Biological Safety Cabinet (BSC) Class II and within a clean room with ISO 5 equivalent or above air quality. In summary, lipoaspirate was separated into stromal vascular fraction using enzymatic digestion and centrifugation and later cell culturing performed under hypoxic conditions within standard growth media containing $10 \%$ fetal bovine serum (FBS). Cells were cultured until $80 \%$ confluency was reached and further cultured until passage 2 (P2). At completion of P2, cells were washed three times to remove FBS and cryopreserved using a validated control rate freezing method $[42,43]$.

Characterization of isolated ADMSCs was confirmed using international established standards as published by the International Society of Cellular Therapy [44]. Flow Cytometry Fluorescent Activated Cell Sorting (FACS) analysis was completed independently on all isolated ADMSC samples at Monash University. Samples were tested for the presence of MSC surface markers CD90, CD73 and CD 105 and absence of hematopoietic surface markers CD14, CD19, CD34 and CD45 (Table 2 and Supplementary Material). All isolated ADMSC samples underwent additional independent sterility testing for microbial growth/contamination.

\section{Injection method}

On the day of injection stored cells were thawed using a sterile water bath with cyroprotectant removed under centrifugation and washed in chilled phosphate buffered saline (PBS). The resultant cell pellet was resuspended in injectable sterile isotonic ( $0.9 \%)$ normal saline to a total of $3 \mathrm{ml}$. Cell count and viability was confirmed using a Muse Cell Analyzer (Merck Millipore, USA; Table 3 and Supplementary Material). For a single participant in the two-injection group the muse cell analyzer was not available for the baseline injection and a manual hemocytometer count was performed.

At the time of injection, the knee of the participants allocated to an active treatment group was prepped using standard sterile protocols. All injections were performed under direct visualization using ultrasound guidance to ensure accuracy. Local anesthetic ( $2 \mathrm{ml}$ of $1 \%$ lidocaine) was infiltrated superficial to the knee joint capsule. Under aseptic conditions, and using a superolateral patella approach, the re-suspended autologous ADMSCs were injected into the intra-articular knee space.

\section{Post-operative rehabilitation}

Participants in the treatment groups were provided with post injection analgesia as required. They were advised to remain nonweight bearing with the use of crutches for 4 weeks. Education regarding range of motion and quadriceps activation exercises was provided. Participants in the two injection group were not required to be non-weight bearing after the second injection at 6 months.

\section{Outcome measures}

Primary outcome aims of this trial were pain and functional changes as measured by the Numeric Pain Rating Scale (NPRS), the Knee Injury and Osteoarthritis Outcome Score and Western Ontario and McMaster Universities 


\begin{tabular}{|lc}
\hline Outcome measure & Measurement point (months) \\
Primary Outcome Measure & $0,1,3,6,12$ \\
1. Numeric Pain Rating Scale & $0,1,3,6,12$ \\
2. Knee Injury and Osteoarthritis Outcome Score & $0,1,3,6,12$ \\
3. Western Ontario and McMaster Universities Osteoarthritis Index & 0,12 \\
Secondary Outcome Measure & \\
1. MRI analysis & \\
- MRI Osteoarthritis Knee Score & \\
\hline
\end{tabular}

Osteoarthritis Index (WOMAC). Secondary outcomes included structural assessment through use of MRI and additional outcome questionnaires (see Table 4).

\section{Questionnaires}

Outcome questionnaires were recorded using the software program Clinical Intelligence (Clinical Intelligence, Melbourne, Australia) and were recorded at baseline, 1, 3, 6 and 12 months.

- Numeric Pain Rating Scale (NPRS). Participants were asked to rate their average pain intensity over the past week on a scale of $0-10$. The NPRS has been validated for use in people with knee OA [45].

- The Knee Injury and Osteoarthritis Outcome Score (KOOS). The KOOS is a validated score that consists of 5 subscales pain improvement, symptom improvement, activities of daily living, function in sport and recreation and knee-related quality of life. A normalized score is calculated for each subscale with 100 indicating no symptoms and 0 indicating maximal symptoms [46].

- The Western Ontario and McMaster Universities Arthritis Index (WOMAC Index 3.0). A validated quality of life score, the WOMAC score quantitatively assesses pain, stiffness and physical function in patients with symptomatic OA [47]. In this trial the WOMAC score is presented as an inverse percentage to be more easily compared with the KOOS subscales where 100 indicates no symptoms.

\section{Magnetic resonance imaging}

Magnetic resonance imaging (MRI) offers the ability to perform a non-invasive evaluation of structural changes associated with OA. The MRI Osteoarthritis Knee Score (MOAKS) is a validated semi-quantitative tool for the evaluation of knee OA [48]. The knee is divided into 14 sub-regional divisions (patella - medial and lateral; femoral - medial and lateral trochlea/central/posterior; tibia - medial and lateral, anterior/central/posterior) with each region achieving a score for features of OA. Features assessed for the purpose of this study were:

- Bone marrow lesions (BMLs) and cysts. Each region is graded for the percentage of volume of each BML and whether there is an associated cyst.

- Articular cartilage. Each region is graded on the percentage area of cartilage with partial to full thickness loss and also separately on area with full thickness loss.

- Osteophytes. Size of osteophytes in 14 sub-regions are graded as Grade 0 (none), Grade 1 (small), Grade 2 (medium) and Grade 3 (large).

- Synovitis. Volume of effusion-synovitis is graded as Grade 0 (none), Grade 1 (small), Grade 2 (medium) and Grade 3 (large).

- Meniscus. Medial and lateral meniscus are assessed for signal and morphology of the tear including vertical, radial, horizontal, complex, root tear, partial or complete maceration.

- Peri-articular features. Popliteal cyst - scored as present or not present.

MOAKs scores were used to describe OA progression over time as previously published [49]. Subregional scores were collated into the broader regions of medial, lateral and patella-femoral compartments.

MRI analysis was performed on a $1.5 \mathrm{~T}$ or greater MRI system with a dedicated peripheral knee coil. Proton density (PD) and proton density fat saturated (PDFS) images were completed in coronal, sagittal and axial planes.

MRI assessment was conducted by a musculoskeletal physician trained in MRI analysis using a single blinded approach with no knowledge of the treatment group. 


\section{Sample size}

To date there is insufficient published data on the effects of autologous MSC therapy in OA. As such, sample size calculations are not feasible. This study represents a pilot RCT to assess treatment protocols for safety and efficacy prior to development of a larger RCT and is consistent with previously published study guidelines [50].

\section{Randomization \& allocation}

Participant group allocation was prepared in advance using a web-based automated random number generator (www.randomizer.org). This was prepared by a designated researcher not involved in the recruitment, screening, assessment, enrolment or treatment process. All researchers with direct involvement in participant recruitment and treatment were blinded to this process.

\section{Adverse events}

Adverse events (AEs) were defined as an undesirable clinical development in a participant which was not present at baseline or which increased in severity after treatment. AEs were assessed and graded in regards to severity:

- Mild: awareness of a sign or symptom which does not interfere with the participants' usual daily activity and/or is transient, resolving with use of simple interventions including simple analgesia.

- Moderate: interferes with the participants' usual daily activity and/or requires symptomatic treatment including regular analgesia (i.e., opiate analgesia).

- Severe: symptom(s) causing severe discomfort with significant impact of the participants' usual daily activity.

- Serious: any unexpected medical incident which requires hospitalization, results in long term disability, is life-threatening or results in death.

\section{Statistical analysis}

SigmaPlot 10 graphical and statistical software (Systat Software, Inc., CA, USA) was used to conduct the analysis. Two Way Repeated Measures Anova (One Factor Repetition, General Linear Model) was utilized to detect mean differences in the independent variables of Treatment (two injections, one injection or control) and Time (baseline, 1, 3, 6 and 12 months) for the dependent variables of NPRS, WOMAC and KOOS scores.

The objectives of the data analysis were first to determine if differences in means occurred between treatments at each of the 5 time points, and second, to determine if mean differences occurred within each of the three groups when comparing 1, 3, 6 and 12 months data to baseline measurements.

When statistically significant $(\mathrm{p}<0.05)$ interactions between the factors of Treatment and Time were apparent an All Pairwise Multiple Comparison Procedure (Holm-Sidak method) was employed to determine the specific mean differences for both Time and Treatment factors with alpha again set at 0.05 .

For the AEs and MOAKs categorical data analysis, Chi-square tests were performed. For the MOAKs data analysis the percentage data was first converted to categories (yes or no) so as to perform the Chi-square tests. Note that due to low sample sizes and low numbers in each category these tests were typically below a power level of 5 .

\section{Patient \& public involvement}

Patients and/or the public were not involved in the development of the research question, choice of outcome measures, design of the trial, recruitment of participants and conduct of the trial. Results of the trial will be disseminated to study participants through direct consultation with a trial clinician at completion of the trial and on publication of results.

\section{Results}

Intergroup comparison \& demographics

As indicated in the Figure 1 flow chart, two participants (one in the Control Group and also another in the two-injection group) were excluded from radiological follow-up analysis as there was significant delay in follow-up MRI (>3 months). One participant was excluded from questionnaire analysis due to incomplete questionnaire data collection (though completed radiological follow-up).

Demographics of the study participants are presented in Table 5. Despite randomization there was a difference in body mass index (BMI) between groups with the control group being, on average, overweight, while both treatment groups were within the obese range. 


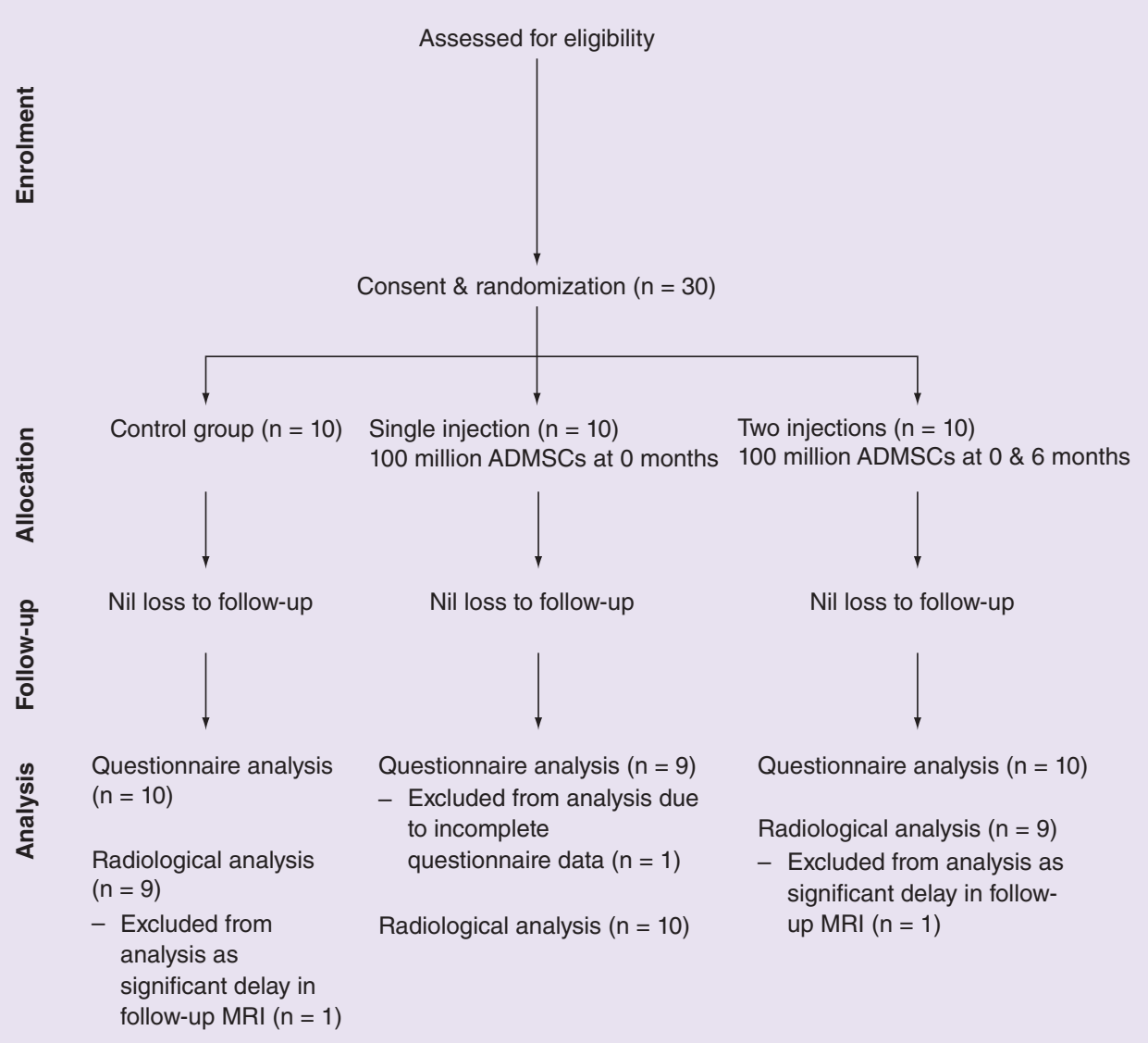

Figure 1. Flow chart.

ADMSC: Adipose-derived mesenchymal stem cells; MRI: Magnetic resonance imaging.

Table 5. Demographic characteristics for the Control and Intervention Groups. Mean data are presented with standard deviations in brackets. Note the significant difference $(p<0.05)$ for body mass index with the one-injection group having a significantly higher body mass index than the Control group.

\begin{tabular}{|c|c|c|c|c|}
\hline Demographic Characteristics & Control & One injection & Two injections & p-value \\
\hline Total number $(\mathrm{n})$ & 10 & 10 & 10 & \\
\hline Gender ( $\%$ male) & 50 & 70 & 40 & \\
\hline Age (years) & $51.5(6.1)$ & $54.6(6.3)$ & $54.7(10.2)$ & 0.58 \\
\hline Height (m) & $1.73(0.1)$ & $1.75(0.1)$ & $1.7(0.1)$ & 0.59 \\
\hline Weight (kg) & $76(14.5)$ & $97.1(20.2)$ & $88.7(23.5)$ & 0.07 \\
\hline BMI $\left(\mathrm{kg} / \mathrm{m}^{2}\right)$ & $25.2(3.4)$ & $31.6(5.9)$ & $30.4(5.6)$ & 0.023 \\
\hline
\end{tabular}

\section{Pain \& functional outcome measures}

Pain, as measured by the NPRS, in the one-injection group and two-injection group improved from a mean (standard deviation) of 6.7 (1.7) and 6.5 (1.4) to 2.6 (1.8) and 2.3 (2) respectively at completion of the study. Within group improvement when compared with baseline was statistically significant $(<0.05)$ throughout all time points in both the single and two injection protocol treatment groups (Figure 2). Similarly, between group differences revealed that both the one-injection and two-injection groups had significantly less pain at 12 months compared with the Control group (Figure 2). No significant differences were found between the two treatment groups.

KOOS subscale analysis showed consistent improvement in all subscales during follow-up and this was maintained until completion of follow-up at 12 months. There was no significant difference at 12 months in all subscales 


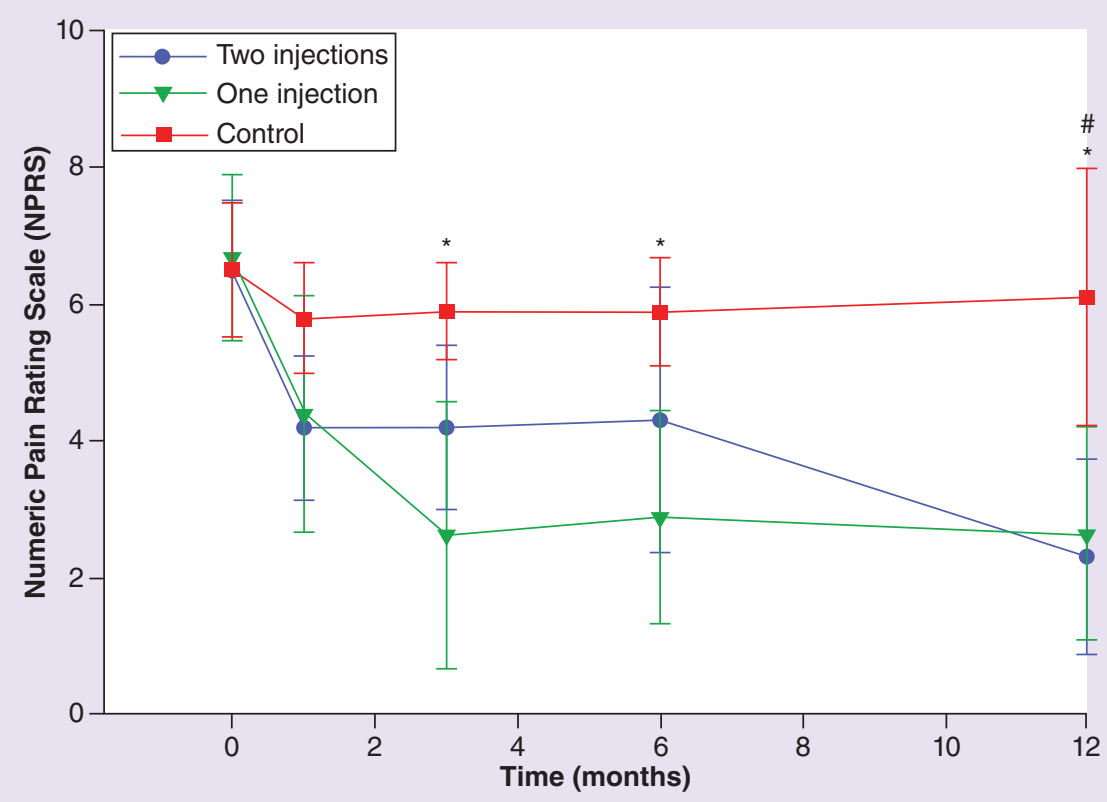

Figure 2. Numeric pain rating scale.

*indicates a significant difference between the one-injection group and the Control group at that time point.

\#represents a significant difference between the two-injection group and the Control group at that time point. Error bars represent $95 \%$ confidence intervals. Within-group differences (not displayed) are detailed in preceding text and are presented in the Supplementary Table data.

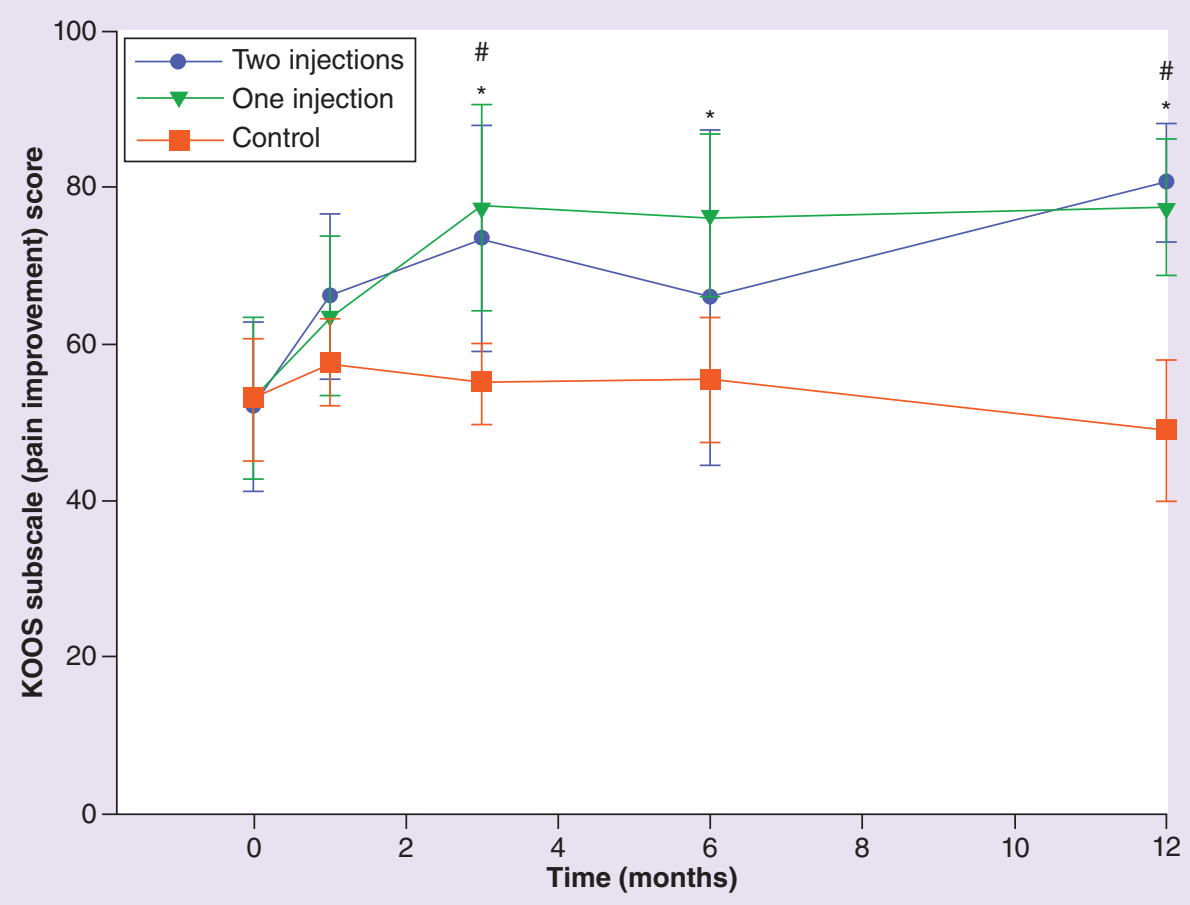

Figure 3. Knee injury and osteoarthritis outcome score pain improvement subscale.

*indicates a significant difference between the one-injection group and the Control group at that time point.

\# represents a significant difference between the two-injection group and the Control group at that time point. Error bars represent $95 \%$ confidence intervals. Within group differences presented in the Supplementary Table data. 


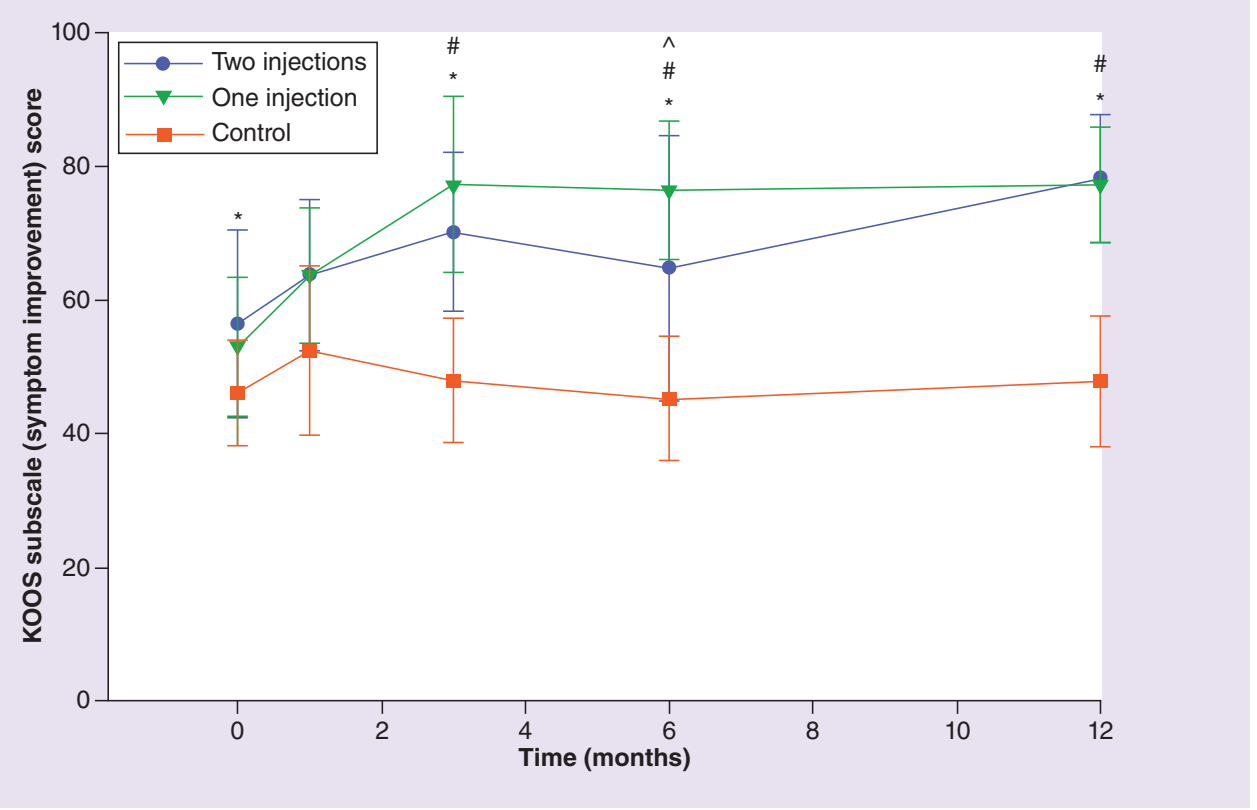

Figure 4. Knee injury and osteoarthritis outcome score pain improvement subscale.

*indicates a significant difference between the one injection group and the Control group at that time point.

\# represents a significant difference between the two-injection group and the Control group at that time point. $\wedge$ indicates a significant difference between treatment groups. Error bars represent $95 \%$ confidence intervals. Within group differences presented in the Supplementary Table data.

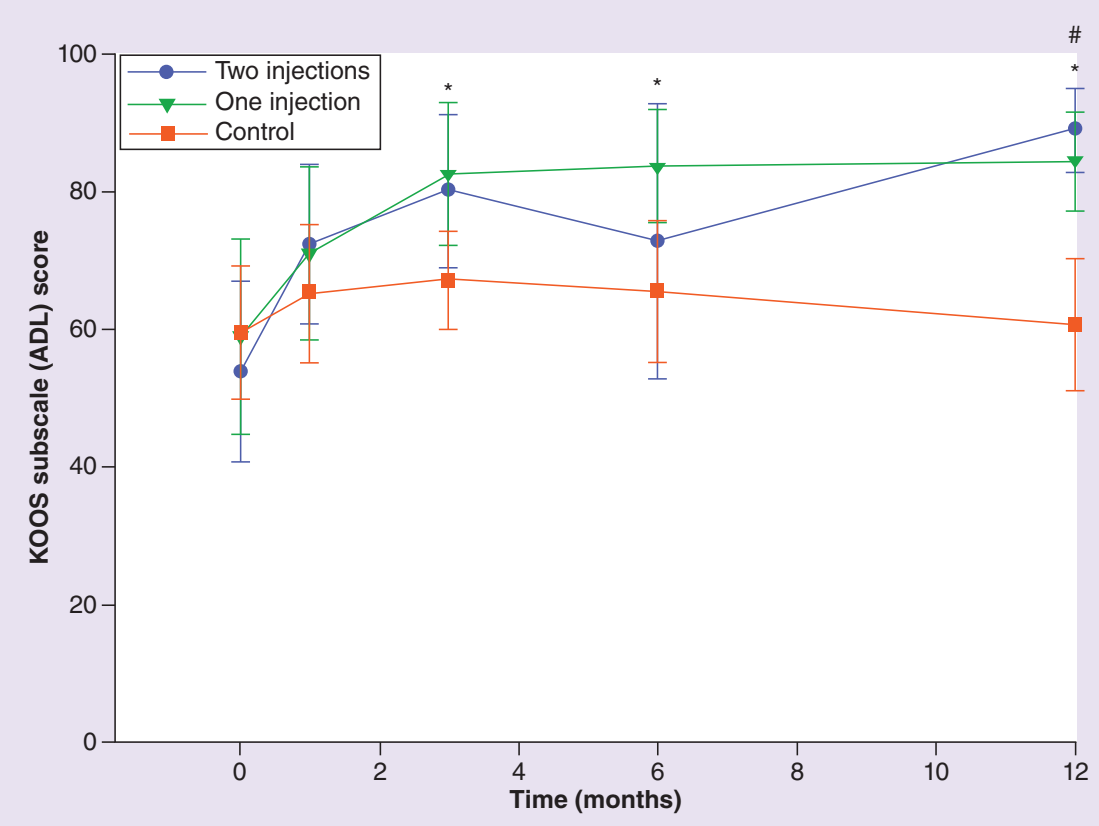

Figure 5. Knee injury and osteoarthritis outcome score Activities of Daily Living subscale.

*indicates a significant difference between the one-injection group and the Control group at that time point.

\# represents a significant difference between the two-injection group and the Control group at that time point. Error bars represent $95 \%$ confidence intervals. Within-group differences presented in the Supplementary Table data. ADL: Activities of daily living; KOOS: Knee injury and osteoarthritis outcome score. 


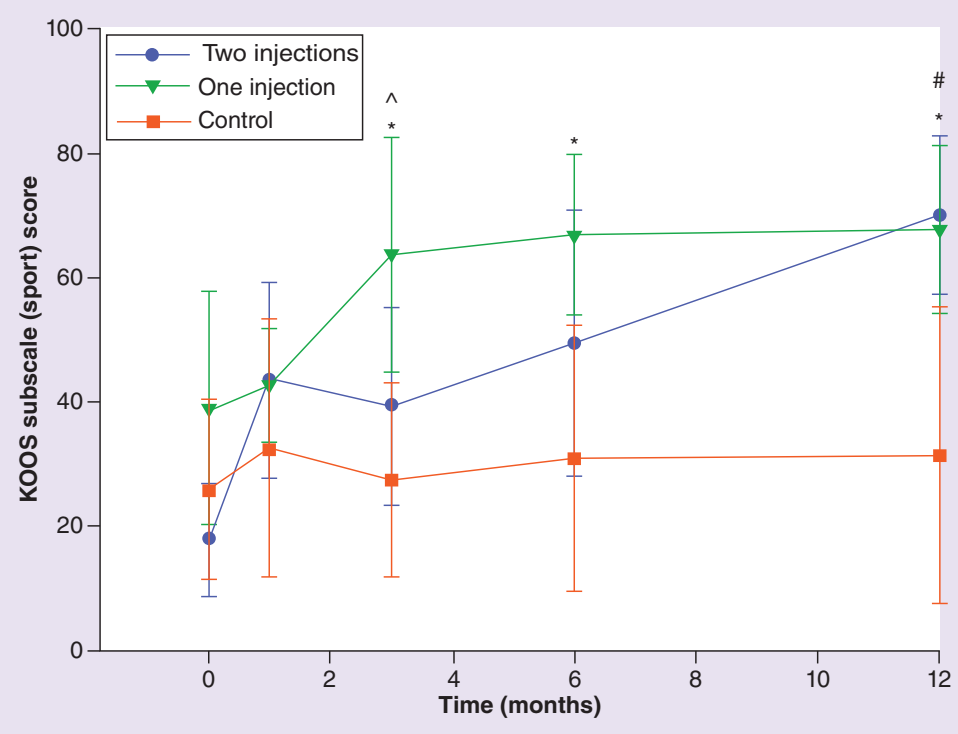

Figure 6. Knee injury and osteoarthritis outcome score sport and recreation subscale.

*indicates a significant difference between the one-injection group and the Control group at that time point.

\# represents a significant difference between the two-injection group and the Control group at that time point. $\wedge$ indicates a significant difference between treatment groups at that time point. Error bars represent $95 \%$ confidence intervals. Within-group differences presented in the Supplementary Table data.

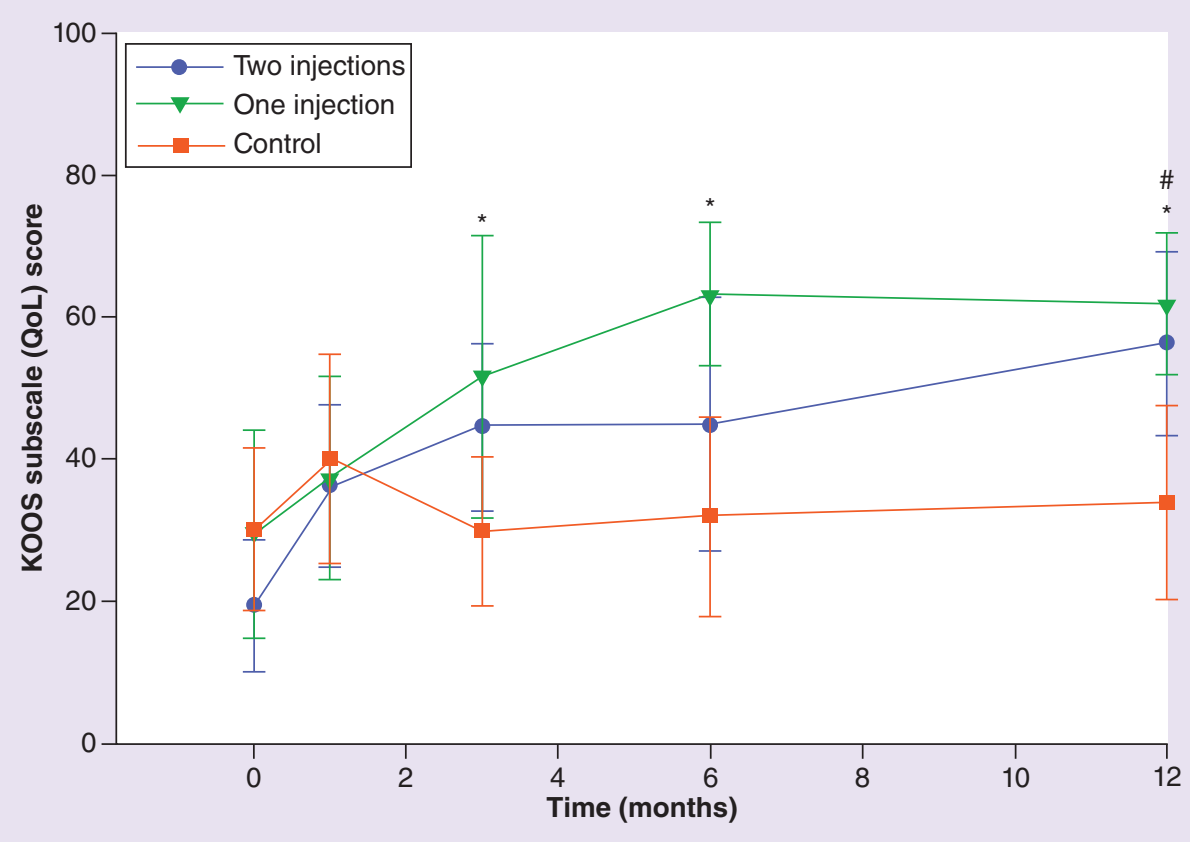

Figure 7. Knee injury and osteoarthritis outcome score Quality of Life subscale.

*indicates a significant difference between the one-injection group and the Control group at that time point.

\# represents a significant difference between the two-injection group and the Control group at that time point. Error bars represent $95 \%$ confidence intervals. Within-group differences presented in the Supplementary Table data. KOOS: Knee Injury and Osteoarthritis Outcome Score; QoL: Quality of life. 
between the two treatment groups. All subscales displayed statistically significant improvement against control at 12 months of follow-up. The one-injection group had more consistent improvement against Control throughout the follow-up period. See Figures 3-7.

Global WOMAC score expressed as an inverse percentage improved throughout follow-up in both the oneinjection group and two-injection group with a mean (standard deviation) of 59.6 (17.9) and 54.4 (18.2) at baseline improving to $84(9.4)$ and 87.3 (8) at 12 months. Statistically significant improvement versus baseline measures was observed as early as 1 month and until final follow-up at 12 months. Both treatment groups had statistically significant improvement at 12 months in comparison to control (Figure 8).

The mean power for all statistical tests performed for pain and functional outcome measures $(\mathrm{n}=21)$ was $0.877 \pm 15$ SD.

Importantly, the percentage of participants achieving a minimal clinically important difference, when combining all pain and functional outcome measures, was 84.1 and $87.1 \%$ in the 1 injection and two-injection groups, respectively (Table 6). Age and gender were not found to influence response to therapy.

See Supplementary Material for tabulated NPRS, KOOS and WOMAC results.

\section{Structural analysis}

MRI Osteoarthritis Knee Scores (MOAKS) were completed at baseline and at 12 months follow-up. One participant in the control group and one participant in the two-injection group were disregarded from MRI analysis due to considerable delay in 12 month imaging. Table 7 indicates the baseline prevalence and change in Global MOAKS at 12 months follow-up of the control and treatment groups (see Supplementary Material for regional results). A total of $67 \%$ of participants within the control group had progression of cartilage loss with a further $56 \%$ having extension of osteophyte formation. By comparison, in the one-injection group only $30 \%$ of participants had further cartilage loss although $50 \%$ had progression of osteophyte formation at 12 months. In the two-injection group $89 \%$ of participants had improvement in cartilage or no progression in cartilage loss with stabilization of OA also indicated by $89 \%$ having no progression in osteophyte formation. The two-injection group was the only group to

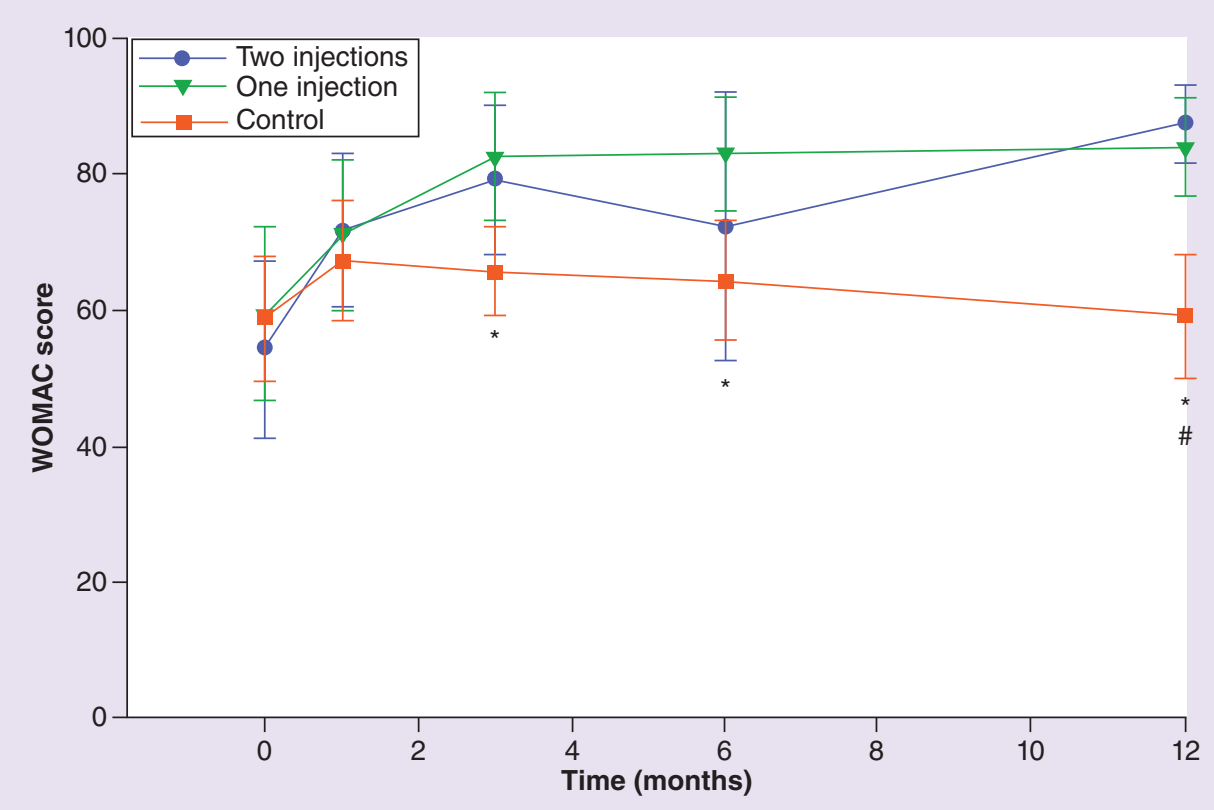

Figure 8. Global Western Ontario and McMaster Universities arthritis index score.

*indicates a significant difference between the one-injection group and the Control group at that time point.

\#represents a significant difference between the two-injection group and the Control group at that time point. Error bars represent $95 \%$ confidence intervals. Within group significant differences were at 3,6 and 12 months compared with baseline for the one-injection group and at 1, 3, 6 and 12 months for the two injection group when compared with baseline (see Supplementary Table Data). 


\begin{tabular}{llll} 
Table 6. Minimal Clinically Important Difference at & $\mathbf{2}$ months in comparison to Baseline data for the three groups. \\
\hline Outcome measures & Control Group & One-injection group & Two-injection group \\
\hline NPRS & 40 & 87.5 & 100 \\
\hline WOMAC & 20 & 100 & 90 \\
\hline KOOS (pain improvement) & 10 & 90 & 80 \\
\hline KOOS (symptom improvement) & 30 & 66.7 & 70 \\
\hline KOOS (ADL) & 30 & 77.8 & 90 \\
\hline KOOS (Sport \& Rec) & 30 & 77.8 & 100 \\
\hline KOOS (QoL) & 20 & 88.9 & 80 \\
\hline Averages & $25.7 \%$ & $84.1 \%$ & $87.1 \%$ \\
\hline A
\end{tabular}

All data are in percentages and expressed as the percentage of subjects who registered a MCID at 12 months when compared with their baseline value. Note that an MCID equates to a decrease of 1 point or more on a 10 point Numeric Pain Rating Scale and an increase of 8 points or more on a 100 point Knee Injury and Osteoarthritis Outcome Score or Western Ontario and McMaster Universities Arthritis Index score.

ADL: Activities of daily living; KOOS: Knee Injury and Osteoarthritis Outcome Score; NPRS: Numeric Pain Rating Scale; WOMAC: Western Ontario and McMaster Universities Arthritis Index.

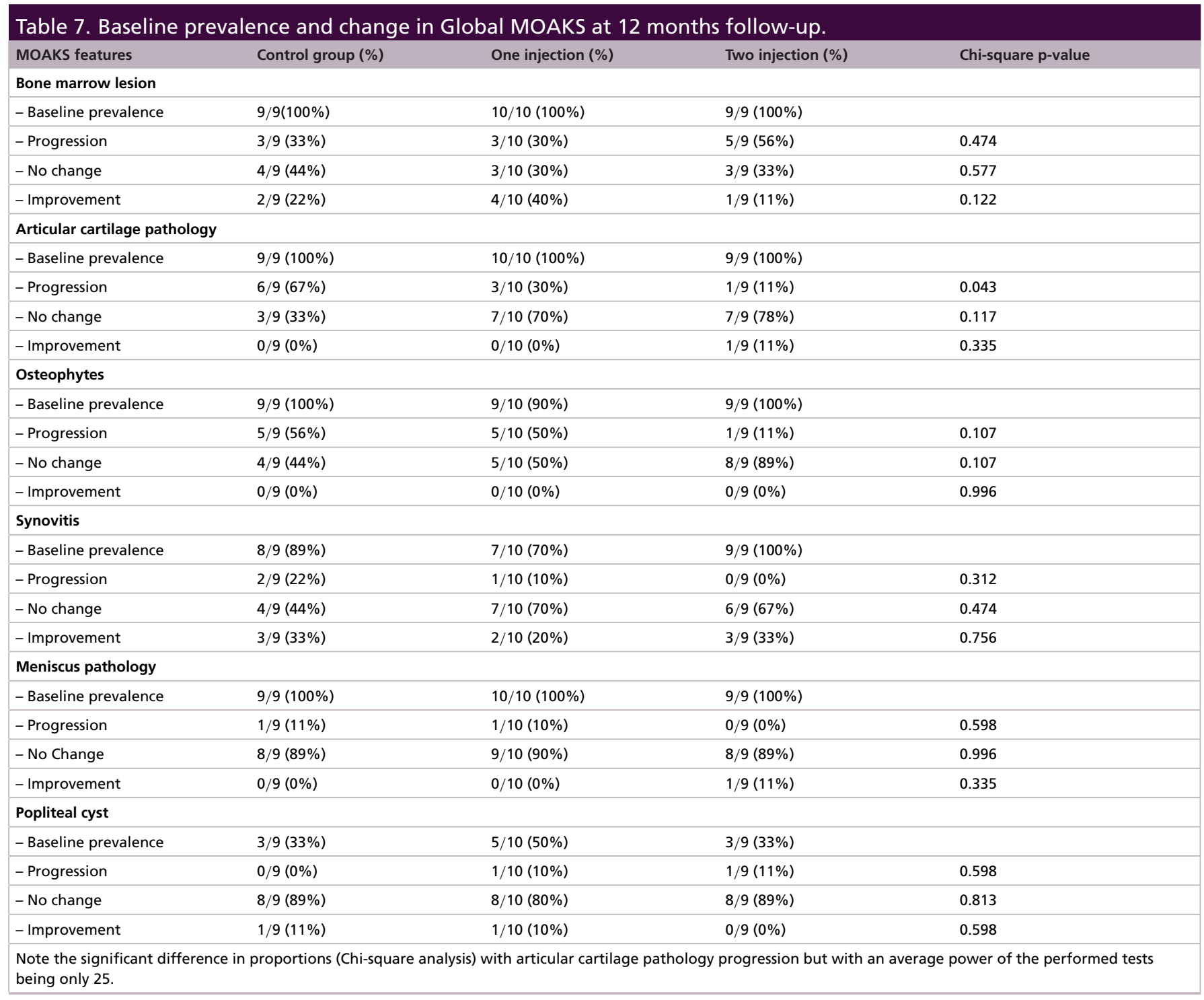


Table 8. Adverse events for the one- and two-injection treatment groups. Note that no significant difference in the proportions (Chi-square analysis) of adverse events between groups were apparent $(p=0.303)$ with the power of the performed test being 0.47 .

\begin{tabular}{|llll|}
\hline Adverse events & One injection (baseline; $\%)$ & Two injections (baseline injection; \%) & Two injections (6 month injection; \%) \\
\hline Nil & $20 \%$ & $10 \%$ & $0 \%$ \\
\hline Mild & $60 \%$ & $50 \%$ & $40 \%$ \\
\hline Moderate & $10 \%$ & $30 \%$ & $60 \%$ \\
\hline Severe & $10 \%$ & $10 \%$ & $0 \%$ \\
\hline Serious & $0 \%$ & $0 \%$ & $0 \%$ \\
\hline
\end{tabular}

show improvement in cartilage loss based on MOAKS analysis (a single participant). Bone marrow lesions (BMLs), synovitis, meniscus pathology or popliteal cysts showed no appreciable difference between groups.

\section{Complications \& adverse events}

Minor discomfort and bruising was commonly noted in both treatment groups after their lipoharvest procedure. This resolved without further intervention indicating a mild expected AE. Table 8 indicates AEs associated with the intra-articular injections of autologous ADMSC therapy at baseline and also at 6 months in the two-injection group. Many participants in the treatment groups experienced discomfort and/or swelling post ADMSC therapy. These AEs were self-limiting, requiring a period of unloading, analgesia and/or oral anti-inflammatory use. Two participants reported pain and swelling for 4 weeks following ADMSC therapy and due to observed impact on their usual daily activity this was categorised as a severe AE. The second injection of MSC therapy at 6 months in the two-injection group was associated with a modest increase in reported moderate AEs in comparison to the initial injection. No related serious AEs were observed.

\section{Discussion}

Previous studies have indicated the potential benefit of MSC therapy in symptomatic knee OA. It is important to differentiate the results of this trial with past trials using more heterogeneous cell based products including stromal vascular fraction [51,52]. This study is the first to assess and compare the safety and efficacy of different treatment protocols with single versus multiple injections and using autologous isolated and expanded ADMSCs. The results indicate that autologous MSC therapy is a safe and effective treatment modality for moderate knee OA with the ability to modify disease progression.

Pain and functional improvement was comparable in both treatment groups. Both statistical and clinically significant change (MCID) was observed in comparison to both baseline and the control group using the NPRS, WOMAC and KOOS subscale scores. The two injection group had an observed mild reduction in improvement in KOOS subscale scores at 6 months although this was as a direct consequence of outcome questionnaires being completed by some participants in the period directly after their second injection (which was associated with a self-limiting period of pain and swelling). Despite some studies indicating stem cell age may be associated with reduced function, this trial did not observe any age-related difference in pain and functional outcome [53].

Pain and functional improvements observed in this pilot RCT compared favorably against conventional treatments for OA. NPRS improved by a percentage of $69 \%$ from baseline to final follow-up at 12 months in both treatment groups. This is in comparison to a $<14 \%$ improvement in pain scores at 12 months following arthroscopic debridement or up to $12 \%$ improvement with a prescribed exercise program [54,55].

Past clinical trials had indicated the ability of intra-articular MSC therapy to modify the progression of OA. Jo and colleagues observed increased cartilage volume at 6 months following MSC therapy, with a later study using allogeneic bone marrow derived MSCs successfully stabilizing OA and improving observed cartilage quality [33,35]. Using the semi-quantitative and validated MOAKS assessment to describe structural change over 12 months, the two injection protocol was observed to achieve a trend of greater disease stabilization in comparison to the single injection group. Observed stabilization rather than cartilage regrowth suggests that the modality of action of MSCs is via paracrine and local supportive pathways rather than direct differentiation along a chondrocyte lineage. This is consistent with preclinical trials which have failed to show significant incorporation of labeled MSCs into regenerative cartilage tissue [28]. 
Surprisingly, features including BMLs, synovitis and the presence of a popliteal cyst were not associated with pain and functional outcome. Several participants in both treatment groups exhibited clinically significant pain improvement despite no change or in fact progression in BMLs. The lack of correlation of improvement with BMLs and synovitis/effusion is in contrast to previous studies indicating these features to be strongly associated with pain in $\mathrm{OA}[56,57]$. This perhaps reflects the observation of lack of improvement in knee pain and function following treatment with bisphosphonates (residronate) in previous RCTs [58,59]. Furthermore, MOAKs analysis indicating successful stabilization despite persistent BMLs is also in contrast to past research which has shown an association between BMLs and progression in OA [60].

Both treatment protocols were well tolerated with no serious AE documented from either the stem cell harvest technique (liposuction) or ADMSC therapy. Whilst two participants experienced pain and swelling, which was classed as a severe $\mathrm{AE}$, this was managed appropriately with a period of unloading and oral analgesia/antiinflammatories and resolved without further intervention required. All AEs were expected and similar to past adverse outcomes reported in previous trials using intra-articular MSC therapy. A modest increase in reported moderate AEs at the second injection in the two-injection group suggests that timing of repeat MSC therapy may influence therapy tolerability.

The main limitation in this RCT is lack of a formal placebo with all groups unblinded to treatment. With the requirement of a lipoharvest procedure to harvest autologous ADMSCs it was deemed unethical to perform an unnecessary surgical procedure on the control group if they were later only to receive a sham placebo injection. This trial represents a pilot study and further research with greater participant numbers is warranted. The MRI analysis is limited in that MOAKS is a semi-quantitative measure. In future research the authors would consider the use of more precise MRI quantitative measures including cartilage volume as described by Cicuttini and colleagues and also the novel and recently validated method of cartilage quality assessment using T2 mapping techniques [61,62].

\section{Conclusion}

$\mathrm{OA}$ is a cause of significant morbidity and with an aging population it promises to remain an enormous community and economic burden. Current conservative management strategies fail to alter disease progression and surgical management in the form of joint replacement is associated with considerable risk. In this RCT, autologous ADMSC therapy was associated with clinically significant improvement in pain and function in symptomatic knee OA. These improvements were well beyond that achieved by conventional methods. Furthermore, ADMSCs were associated with successful disease modification with comparison of treatment protocols indicating that two intra-articular injections of ADMSCs separated by 6 months achieved more consistent OA stabilization than a single injection. Intra- articular autologous ADMSC therapy was shown to be safe with no serious AEs.

\section{Translational perspective}

Regenerative cellular therapies rather than being unique and novel are now well-established and form part of accepted medical practice in areas including bone marrow and tissue transplantation, blood transfusion and reproductive in vitro fertilization. First described by Dr Alexander Friedenstein over 40 years ago, MSCs, with their observed multipotency and presence throughout the body, have been postulated as a future biological cell therapy for tissue repair and regeneration. Recent years have seen a significant increase in the number of clinical trials involving MSCs with international groups including the International Society for Stem Cell Research publishing guidelines for appropriate stem cell research and clinical translation [63]. An acknowledgment of the various different cellular therapies being both studied and used clinically in the area of musculoskeletal medicine, and yet often described in broad inclusive terminology, has seen the development of a minimum information for studies evaluating biologics in orthopedics (MIBO) [64].

This trial represents the first published RCT on the use of autologous isolated and expanded ADMSCs in OA. Significant pain and functional improvement and observed disease modification with structural stabilization indicate that ADMSC therapy may represent an exciting advancement in the treatment of OA. Additional research with direct comparison against accepted conventional treatment options needs to be conducted. 
Summary points

- Osteoarthritis (OA) is the fourth leading cause of disability worldwide.

- Symptomatic arthritis affects $10 \%$ of males and $18 \%$ of females over the age of 45 years.

- $80 \%$ of patients over the age of 65 have radiological evidence of OA.

- Current conservative management strategies are aimed at symptom control and do not modify the progression of OA. Pharmaceutical treatments are limited and may have serious side effects.

- Surgical total joint replacement remains the accepted treatment for knee OA uncontrolled by conservative therapies and is not without complication with up to $20 \%$ of patients experiencing persistent pain and loss of function at 12 months.

- Initial preclinical and clinical trials have indicated the ability of intra-articular mesenchymal stem cel therapies to improve pain and function.

- This is the first randomized controlled trial to assess the safety and efficacy of autologous isolated and expanded adipose derived mesenchymal stem cell (ADMSC) therapy in symptomatic knee OA.

- Over 12 months of follow-up statistical and importantly clinically significant pain and functional improvements were observed in both treatment groups against control and baseline values as measured by validated outcome scores including Numeric Pain Rating Scale, Western Ontario and McMaster Universities Arthritis Index and Knee Injury and Osteoarthritis Outcome Score.

- Semi-quantitative radiological analysis using the magnetic resonance imaging based Osteoarthritis Knee Score assessment indicated modification of disease progression over 12 months of follow-up with greatest stabilization achieved by the treatment group receiving two injections of ADMSCs.

- No serious adverse events were observed.

- Improvement in pain scores compared favorably to previous studies on the effectiveness of current conventional therapies.

- Autologous ADMSC therapy represents a potential exciting advancement in the active management of OA.

\section{Supplementary data}

To view the supplementary data that accompany this paper please visit the journal website at: www.futuremedicine.com/doi/full/ 10.2217/rme-2018-0161

Financial \& competing interests disclosure

J Freitag, D Bates, L Huguenin, A Tenen are clinic partners within Melbourne Stem Cell Centre.

J Freitag, D Bates, R Boyd, K Shah, L Huguenin, A Tenen are associated with Magellan Stem Cells and are part of its Medical and Scientific Advisory Committee.

This proposed study was funded jointly by both Magellan Stem Cells and Melbourne Stem Cell Centre. Members of their Medical and Scientific Advisory board have been involved in the study conception and design and are listed as co-authors of this paper. The authors received no payment for their involvement in the study. Interpretation of results, and subsequent submission and publication decisions have been made independent of the sponsors.

The authors have no other relevant affiliations or financial involvement with any organization or entity with a financial interest in or financial conflict with the subject matter or materials discussed in the manuscript apart from those disclosed.

Author contributions

J Freitag, D Bates, R Boyd, K Shah, L Huguenin, A Tenen, K Paterson were involved in conception of the study. J Freitag, D Bates, J Wickham, K Shah, R Boyd, L Huguenin, A Tenen, K Paterson designed the study protocol. J Wickham was involved in developing the statistical analysis strategy.

\section{Acknowledgments}

The authors would like to acknowledge the work of biostatistician S Nielsen for statistical analysis review.

The authors would like to acknowledge the following people for assistance in project management:

R Castelluccio - Nurse Project Manager.

M Grogan and E Picken - Study Co-ordinators.

N Shah, I Wickramasinghe and M Lopez - Laboratory Staff. 


\section{Ethical conduct of research}

Ethics approval was given for the study by the Human Research Ethics Committee of Monash University and registered on the Australian and New Zealand Clinic Trial Registry (Trial ID: ACTRN12614000814673). In addition, informed consent has been obtained from the participants involved.

\section{Data sharing statement}

Individual de-identified participant data which underlie results reported in this article will be available upon publication. The study protocol will also be available. Material will be accessible to investigators whose proposed use of data has been approved by an independent review committee and for data meta-analysis. Requests are to be directed to the corresponding author of the article.

\section{Open access}

This work is licensed under the Attribution-NonCommercial-NoDerivatives 4.0 Unported License. To view a copy of this license, visit http://creativecommons.org/licenses/by-nc-nd/4.0/

\section{References}

1. Brooks PM. Impact of osteoarthritis on individuals and society: how much disability? Social consequences and health economic implications. Curr. Opin. Rheumatol. 14(5), 573-577 (2002).

2. Fransen M, Bridgett L, March L. The epidemiology of osteoarthritis in Asia. Int. J. Rheum. Dis. 14(2), 113-121 (2011).

3. Gupta S, Hawker GA, Laporte A, Croxford R, Coyte PC. The economic burden of disabling hip and knee osteoarthritis (OA) from the perspective of individuals living with this condition. Rheumatology (Oxford) 44(12), 1531-1537 (2005).

4. Issa SN, Sharma L. Epidemiology of osteoarthritis: an update. Curr. Rheum. Rep. 8(1), 7-15 (2006).

5. Peat G, McCarney R, Croft P. Knee pain and osteoarthritis in older adults: a review of community burden and current use of primary health care. Ann. Rheum. Dis. 60(2), 91-97 (2001).

6. Zhou Q, Yang W, Chen J, Wang Y. Metabolic syndrome meets osteoarthritis. Nat. Rev. Rheumatol. 8, 729-737 (2012).

7. Abraham NS, El-Serag HB. Cyclooxygenase-2 selectivity of non-steroidal anti-inflammatory drugs and the risk of myocardial infarction and cerebrovascular accident. Aliment. Pharmacol. Ther. 25(8), 913-924 (2007).

8. Bagga H, Burkhardt D. Long-term effects of intra-articular hyaluronan on synovial fluid in osteoarthritis of the knee. J. Rheumatol. 33(5), 946-950 (2006).

9. Hawker GA, Mian S, Bednis K, Stanaitis I. Osteoarthritis year 2010 in review: non-pharmacologic therapy. Osteoarthr. Cartil. 19(4), 366-374 (2011).

10. McAlindon TE, Bannuru RR, Sullivan MC et al. OARSI guidelines for the non-surgical management of knee osteoarthritis. Osteoarthr. Cartil. 22(3), 363-388 (2014).

11. Bourne RB, Chesworth BM. Patient satisfaction after total knee arthroplasty: who is satisfied and who is not? Clin. Orthop. Relat 468, 57-63 (2010).

12. Singh JA, Kundukulam J. Early postoperative mortality following joint arthroplasty: a systematic review. J. Rheumatol. 38, 1507-1513 (2011).

13. Wylde V, Hewlett S, Learmonth ID, Dieppe P. Persistent pain after joint replacement: prevalence, sensory qualities, and postoperative determinants. Pain 152(3), 566-572 (2011)

14. Goldring MB. Osteoarthritis and cartilage: the role of cytokines. Curr. Rheumatol. Rep. 2(6), 459-465 (2000).

15. Loeser R. Aging and osteoarthritis: the role of chondrocyte senescence and aging changes in the cartilage matrix. Osteo. Cart. 17, 971-979 (2009).

16. Mitchell PG, Magna HA, Reeves LM. Cloning, expression, and Type II collagenolytic activity of matrix metalloproteinase-13 from human osteoarthritic cartilage. J. Clin. Invest. 97, 761-768 (1996).

17. Arinzeh TL. Mesenchymal stem cells for bone repair: preclinical studies and potential orthopaedic applications. Foot Ankle Clin. 10(4), 651-665 (2005).

18. Barry FP, Murphy JM. Mesenchymal stem cells: clinical applications and biological characterization. Int. J. Biochem. Cell Biol. 36(4), 568-584 (2004).

19. Noel D, Djouad F, Jorgense C. Regenerative medicine through mesenchymal stem cells for bone and cartilage repair. Curr. Opin Investig. Drugs 3(7), 1000-1004 (2002).

20. Caplan A. What are MSCs therapeutic? New data: new insight. J. Pathol. 217, 318-324 (2009).

21. Caplan AI. Mesenchymal stem cells. J. Orth. Res. 9(5), 641-650 (1991).

22. Caplan AI, Correa D. The MSC: an injury drugstore. Cell Stem Cell 9(1), 11-15 (2011) 
23. Djouad F, Bouffi C, Ghannam S. Mesenchymal stem cell: innovative therapeutic tools for rheumatic diseases. Nat. Rev. Rheumatol. 5 , 392-399 (2009).

24. Nakagami H, Morishita R. Adipose tissue-derived stromal cells as a novel option for regenerative cell therapy. J. Atheroscler. Thromb. 13(2), 77 (2006).

25. Caplan AI. Adult mesenchymal stem cells for tissue engineering versus regenerative medicine. J. Cell. Physiol. 213, 341-347 (2007).

26. Black L, Gaynor J, Adams C. Effect of intra-articular injection of autologous adipose-derived mesenchymal stem and regenerative cells on clinical signs of chronic osteoarthritis of the elbow joint in dogs. Vet. Ther. 9, 192-200 (2008).

27. Lee KB, Hui JH, Song IC, Ardany L. Injectable mesenchymal stem cell therapy for large cartilage defects - a porcine model. Stem Cell 25, 2964-2971 (2007).

28. Murphy JM, Fink DJ, Hunziker EB. Stem cell therapy in a caprine model of osteoarthritis. Arthritis Rheum. 48, 3464-3474 (2003).

29. van Buul GM, Siebelt M, Leijs MJC et al. Mesenchymal stem cells reduce pain but not degenerative changes in a mono-iodoacetate rat model of osteoarthritis. J. Orthop. Res. 32(9), 1167-1174 (2014).

30. Centeno C, Busse D, Kisiday J. Increased knee cartilage volume in degenerative joint disease using percutaneously implanted, autologous mesenchymal stem cells. Pain Phys. 11(3), 343-353 (2008).

31. Centeno C, Schultz J, Cheever M. Safety and complications reporting on the re-implantation of culture-expanded mesenchymal stem cells using autologous platelet lysate technique. Curr. Stem Cell 5(1), 81-93 (2011).

32. Jo CH, Lee YG, Shin WH. Intra-articular injection of mesenchymal stem cells for the treatment of osteoarthritis of the knee: a proof of concept clinical trial. Stem Cells 32(5), 1254-1266 (2014).

33. Vega A, Martín-Ferrero MA, Del Canto F et al. Treatment of knee osteoarthritis with allogeneic bone marrow mesenchymal stem cells: a randomized controlled trial. Transplantation 99(8), 1681-1690 (2015).

34. Lalu ML, McIntyre L. Safety of cell therapy with mesenchymal stromal cells (safe cell): a systematic review and meta-analysis of clinical trials. PLoS ONE 7(10), e47559 (2012).

35. Peeters CM, Leijs MJ. Safety of intra-articular cell-therapy with culture-expanded stem cells in humans: a systematic literature review. Osteooarthr. Cartil. 21(10), 1465-1473 (2013).

36. Freitag J, Ford J, Bates D et al. Adipose derived mesenchymal stem cell therapy in the treatment of isolated knee chondral lesions: design of a randomised controlled pilot study comparing arthroscopic microfracture versus arthroscopic microfracture combined with postoperative mesenchymal stem cell injections. BMJ Open 5(12), e009332 (2015).

37. Alvarez-Viejo M, Menendez-Menendez Y, Blanco-Gelaz MA et al. Quantifying mesenchymal stem cells in the mononuclear cell fraction of bone marrow samples obtained for cell therapy. Transplant. Proc. 45(1), 434-439 (2013).

38. Im GI, Shin YW, Lee KB. Do adipose tissue-derived mesenchymal stem cells have the same osteogenic and chondrogenic potential as bone marrow-derived cells? Osteoarthr. Cartil. 13(10), 845-853 (2005).

39. Peng L, Jia Z, Yin X et al. Comparative analysis of mesenchymal stem cells from bone marrow, cartilage, and adipose tissue. Stem Cells Dev. 17(4), 761-774 (2008).

40. Freitag J, Li D, Wickham J, Shah K, Tenen A. Effect of autologous adipose-derived mesenchymal stem cell therapy in the treatment of a post-traumatic chondral defect of the knee. BMJ Case Rep. 2017(bcr2017220852), (2017).

41. Freitag J, Shah K, Wickham J, Boyd R, Tenen A. The effect of autologous adipose derived mesenchymal stem cell therapy in the treatment of a large osteochondral defect of the knee following unsuccessful surgical intervention of osteochondritis dissecans - a case study. BMC Musculoskelet. Disord. 18(1), 298 (2017).

42. Goh BC, Sreedhar T, Gail K, V. DR, M. GJ. Cryopreservation characteristics of adipose-derived stem cells: maintenance of differentiation potential and viability. J. Tissue Eng. Regen. Med. 1(4), 322-324 (2007).

43. Martinello T, Bronzini I, Maccatrozzo L et al. Canine adipose-derived mesenchymal stem cells do not lose stem features after a long-term cryopreservation. Res. Vet. Sci. 91(1), 18-24 (2011).

44. Dominici M, Blanc K. Minimal criteria for defining multipotent mesenchymal stromal cells. The International Society for Cellular Therapy position statement. Cytotherapy 8(4), 315-317 (2006).

45. Dworkin RH, Turk DC, Farrar JT et al. Core outcome measures for chronic pain clinical trials: IMMPACT recommendations. Pain 113(1-2), 9-19 (2005).

46. Roos EM, Roos HP, Lohmander LS, Ekdahl C, Beynnon BD. Knee Injury and Osteoarthritis Outcome Score (KOOS)-development of a self-administered outcome measure. J. Orthop. Sports Phys. Ther. 28(2), 88-96 (1998).

47. Bellamy N, Buchanan WW, Goldsmith CH, Campbell J, Stitt LW. Validation study of WOMAC: a health status instrument for measuring clinically important patient relevant outcomes to antirheumatic drug therapy in patients with osteoarthritis of the hip or knee. J. Rheumatol. 15(12), 1833-1840 (1988).

48. Hunter DJ, Guermazi A, Lo GH et al. Evolution of semi-quantitative whole joint assessment of knee OA: MOAKS (MRI Osteoarthritis Knee Score). Osteoarthr. Cartil. 19(8), 990-1002 (2011). 
49. Runhaar J, Schiphof D, van Meer B, Reijman M, Bierma-Zeinstra SMA, Oei EHG. How to define subregional osteoarthritis progression using semi-quantitative MRI Osteoarthritis Knee Score (MOAKS). Osteoarthr. Cartil. 22(10), 1533-1536 (2014).

50. Moore CG, Carter RE, Nietert PJ, Stewart PW. Recommendations for planning pilot studies in clinical and translational research. Clin. Transl. Sci. 4(5), 332-337 (2011).

51. Centeno C, Kisiday J, Freeman M. Partial regeneration of the human hip via autologous bone marrow nucleated cell transfer: a case study. Pain Phys. 9, 253-256 (2006).

52. Pak J. Regeneration of human bones in hip osteonecrosis and human cartilage in knee osteoarthritis with autologous adipose derived stem cells: a case series. J Med Case Rep 5, 296 (2001).

53. Peffers MJ, Collins J, Fang Y et al. Age-related changes in mesenchymal stem cells identified using a multi-omics approach. European Cells and Materials 31, 136-159 (2016).

54. Moseley JB, O’Malley K, Petersen NJ et al. A controlled trial of arthroscopic surgery for osteoarthritis of the knee. N. Engl. J. Med. 347(2), 81-88 (2002).

55. Thomas KS, Muir KR, Doherty M, Jones AC, O’Reilly SC, Bassey EJ. Home based exercise programme for knee pain and knee osteoarthritis: randomised controlled trial. BMJ 325(7367), 752 (2002).

56. Felson DT, Chaisson CE, Hill CL et al. The association of bone marrow lesions with pain in knee osteoarthritis. Ann. Intern. Med. 134(7), 541-549 (2001).

57. Lo GH, McAlindon TE, Niu J et al. Bone marrow lesions and joint effusion are strongly and independently associated with weight-bearing pain in knee osteoarthritis: data from the osteoarthritis initiative. Osteoarthr. Cartil. 17(12), 1562-1569 (2009).

58. Bingham CO, Buckland-Wright JC, Garnero P et al. Risedronate decreases biochemical markers of cartilage degradation but does not decrease symptoms or slow radiographic progression in patients with medial compartment osteoarthritis of the knee: results of the two-year multinational knee osteoarthritis structural arthritis study. Arthritis Rheum. 54(11), 3494-3507 (2006).

59. Spector TD, Conaghan PG, Buckland-Wright JC et al. Effect of risedronate on joint structure and symptoms of knee osteoarthritis: results of the BRISK randomized, controlled trial. Arthritis Res. Ther. 7(3), R625 (2005).

60. Felson DT, McLaughlin S, Goggins J et al. Bone marrow edema and its relation to progression of knee osteoarthritis. Ann. Intern. Med. 139(5_Part_1), 330-336 (2003).

61. Baum T, Joseph GB, Karampinos DC, Jungmann PM, Link TM, Bauer JS. Cartilage and meniscal T2 relaxation time as non-invasive biomarker for knee osteoarthritis and cartilage repair procedures. Osteoarthr. Cartil. 21(10), 1474-1484 (2013).

62. Cicuttini F, Forbes A, Asbeutah A, Morris K, Stuckey S. Comparison and reproducibility of fast and conventional spoiled gradient-echo magnetic resonance sequences in the determination of knee cartilage volume. J. Orthop. Res. 18(4), 580-584 (2000).

63. ISSCR Task Force. International Society of Stem Cell Research, Guidelines for Stem Cell Research and Clinical

Translation. (2017). www.isscr.org/docs/default-source/all-isscr-guidelines/guidelines-2016/isscr-guidelines-for-stem-cell-research-and - clinical-translationd67119731dff6ddbb37cff0000940c19.pdf?sfvrsn=4

64. Murray IR, Geeslin AG, Goudie EB, Petrigliano FA, LaPrade RF. Minimum information for studies evaluating biologics in orthopaedics (MIBO): Platelet-Rich Plasma and Mesenchymal Stem Cells. JBJS 99(10), 809-819 (2017). 